\title{
Stereotactic body radiotherapy or stereotactic
} ablative radiotherapy versus surgery for patients with TI-3N0M0 non-small cell lung cancer: a systematic review and meta-analysis

\author{
This article was published in the following Dove Press journal: \\ OncoTargets and Therapy \\ 7 June 2017 \\ Number of times this article has been viewed
}

Ming $\mathrm{Li}^{1,2, *}$

Xiaodong Yang ${ }^{1, *}$

Yuhan Chen ${ }^{3}$

Xinyu Yang ${ }^{1,2}$

Xiyu Dai ${ }^{1,2}$

Fenghao Sun'

Li Zhang ${ }^{3}$

Cheng Zhan'

Mingxiang Feng'

Qun Wang'

'Department of Thoracic Surgery, Zhongshan Hospital, ${ }^{2}$ Eight-Year

Program Clinical Medicine, Grade of 2014, Shanghai Medical College,

Zhongshan Hospital, Fudan University,

Shanghai, People's Republic of China

*These authors contributed equally to this work
${ }^{3}$ Department of Radiation Oncology,

Background: Stereotactic body radiotherapy (SBRT) or stereotactic ablative radiotherapy (SABR) has been reported to be a comparable alternative therapy to surgery for patients with T1-3N0M0 non-small cell lung cancer (NSCLC). However, it has not been clarified whether SBRT/SABR is as effective as surgery. We conducted this study to compare the efficacy of SBRT/SABR and surgery in the treatment of T1-3N0M0 NSCLC.

Materials and methods: An electronic and a manual search of the literature was conducted in PubMed, Embase, and the Wiley Online Library in all published data before January 1, 2017. The pooled data included overall survival (OS), recurrence-free survival (RFS), and locoregional/distant recurrence rate. Hazard ratio (HR) of OS (SBRT/SABR vs surgery) was used as the measure of differential effects.

Results: Fifteen studies, including 7,810 patients with T1-3N0M0 NSCLC, 2,986 patients in the SBRT/SABR group, and 4,824 patients in the surgery group, were pooled for the meta-analysis. Results showed that patients with SBRT/SABR had a significantly worse 5-year survival rate ( $\mathrm{HR}=1.40 ; 95 \%$ confidence interval $[\mathrm{CI}]: 1.21,1.61 ; P<0.01)$, and RFS rate (HR $=1.84 ; 95 \%$ CI: $1.26,2.68 ; P=0.002)$. Meanwhile, the locoregional recurrence rate $(\mathrm{HR}=1.17 ; 95 \% \mathrm{CI}$ : $0.68,1.98 ; P=0.57$ ), and distant recurrence rate ( $\mathrm{HR}=1.36 ; 95 \% \mathrm{CI}: 0.77,2.39 ; P=0.29$ ) were also lower in the surgery group although results were not statistically significant. In subgroup analyses, SBRT/SABR had a significantly lower rate of 5-year survival (HR $=1.46 ; 95 \% \mathrm{CI}$ : $1.03,2.06 ; P=0.03)$ compared with lobectomy. Similarly, significant differences of OS exist in comparisons of SBRT/SABR versus sublobectomy (HR $=1.40 ; 95 \% \mathrm{CI}: 1.09,1.80 ; P=0.008)$, and wedge resection ( $\mathrm{HR}=1.48 ; 95 \% \mathrm{CI}: 1.01,2.16 ; P=0.04)$.

Conclusion: Surgery, both lobectomy and sublobectomy, might be superior to SBRT/SABR with regard to survival of patients with T1-3N0M0 NSCLC. Patients with T1-3N0M0 NSCLC should preferably be treated surgically prior to SBRT/SABR.

Keywords: non-small cell lung cancer, stereotactic body radiotherapy, stereotactic ablative radiotherapy, lobectomy, sublobectomy, meta-analysis

\section{Introduction}

Cheng Zhan

Department of Thoracic Surgery,

Zhongshan Hospital, Fudan University,

No 180, Fenglin Road, Shanghai 200032,

People's Republic of China

Tel/fax +86 2I 64041990

Email feng.mingxiang@zs-hospital.sh.cn; czhan10@fudan.edu.cn
Lung cancer is the most frequently diagnosed cancer worldwide. In 2012, an estimated 1.8 million new cases of lung cancer occurred, which accounted for approximately $13 \%$ of total cancer diagnosed. ${ }^{1}$ Non-small cell lung cancer (NSCLC) is the commonest lung malignancy. Surgery, especially lobectomy, was the standard therapy for operable early-clinical-stage NSCLC. ${ }^{2}$ However, for patients with many potential 
comorbidities who are not sufficiently healthy to undergo surgery or due to individual unwillingness, especially in elderly patients, radiotherapy can be considered an alternative option. ${ }^{3}$ Different from traditional radiotherapy, stereotactic body radiotherapy (SBRT), also referred to as stereotactic ablative radiation (SABR), delivers very high radiation doses to restricted volumes using multiple precisely aimed radiotherapy beams; this has a better effect on survival and tumor control and has been the focus of attention in recent years. ${ }^{4}$ Recently, several studies have suggested that, compared with surgery, SBRT/SABR has similar or better survival, together with lower recurrence, for patients with early-stage NSCLC. ${ }^{5-7}$ In the latest published National Comprehensive Cancer Network Clinical Practice Guidelines and the European Society for Medical Oncology Consensus, SBRT/ SABR is recommended as the most favorable therapy choice besides surgery for T1-3N0M0 NSCLC. ${ }^{8}$ There is, however, a paucity of consensus to support SBRT/SABR, and there exist some controversial results and conclusions in this field. Thus, to address this issue, we conducted a systematic review and meta-analysis aiming to compare efficacy and survival outcomes between surgery and SBRT/SABR.

\section{Materials and methods}

We searched PubMed, Embase, and the Wiley Online Library for eligible studies published before January 1, 2017. The following groups of key words or medical terms were used: ("stereotactic body radiotherapy" or "stereotactic ablative radiotherapy" or "SBRT" or "SABR") and ("surgery" or "operation" or "lobectomy" or "sublobar resection" or "limited resection" or "sublobectomy" or "segmentectomy" or "wedge resection") and ("non-small cell lung cancer" or "non-small cell lung carcinoma" or "non-small cell lung neoplasms" or "lung adenocarcinoma" or "lung squamous cell carcinoma" or "large cell lung cancer"). Only articles in English were selected. Furthermore, reference lists of relevant studies were searched for potentially eligible records.

\section{Study inclusion}

The included studies were comparative studies, including both retrospective and prospective studies, that compared radiotherapy, including SBRT/SABR, with surgery, including lobectomy, segmentectomy, or wedge resection, in patients with T1-3N0M0 NSCLC. The clinical stages of NSCLC ranged from I to II. When multiple groups were present in an individual study, we only selected the comparison between lobectomy plus segmentectomy plus wedge resection and SBRT/SABR. In some studies on SBRT/SABR in propensity-matched analysis, we selected the comparison after propensity matching. The outcomes of interest included overall survival (OS), recurrence-free survival (RFS), locoregional recurrence, and distant recurrence. The following studies were excluded: 1) studies not comparing SBRT/ SABR with surgery; 2) case reports, letters, reviews, comments, or meta-analysis; 3) studies lacking necessary data; or 4) overlapped studies or duplicated data.

\section{Data collection and quality assessment}

Two reviewers extracted data using a standardized form independently. When an outcome was followed at different intervals, the one with the longest follow-up was selected. The following information was obtained: author, year, journal, study design, data sources, time span, stage, TNM, tumor size, resection, age, amount, the sources of hazard ratio (HR), 95\% confidence interval [CI], and survival results. HRs and 95\% CIs were obtained from the original article in all cases. To assess the quality of randomized controlled trials (RCTs), we used the Cochrane Handbook for Systematic Reviews of Interventions. We used the modified Newcastle-Ottawa scale (NOS) to evaluate non-randomized controlled studies (NRS). Four aspects were evaluated by the NOS: selection, comparability, exposure, and outcomes. The scores ranged from $0 \pm 7$ points, with $0 \pm 2$ points indicating low quality, $3 \pm 5$ points indicating medium quality, and $>6$ points denoting high quality. ${ }^{9}$

\section{Statistical analysis}

The meta-analysis for comparative outcomes was performed using Review Manager (version 5.3) (http://review-manager. software.informer.com/5.3/). Weighted mean differences with $95 \% \mathrm{CI}$ as the effect estimate and odds ratios (ORs) with 95\% CI were used to analyze continuous data and dichotomous data, respectively. For survival analysis, we extracted data from survival curves by referring to methods reported by Tierney et al, and HRs were used for quantitative analysis. ${ }^{10}$ Heterogeneity was measured using the $P$ index and $P$-value; $P<0.05$ was considered statistically significant; otherwise, no significance was attributed. $I^{2}<25 \%$ was regarded as low heterogeneity, 25\%-75\% was regarded as medium heterogeneity, and $I^{2} \geq 75 \%$ was regarded as high heterogeneity. ${ }^{9}$ A random effect was used when the heterogeneity test had significance; otherwise, a fixed effect was used. Sensitivity analysis was performed by excluding the included studies one by one. Publication bias was assessed with funnel plots.

\section{Results \\ Selection process}

As Figure 1 shows, a total of 721 records were initially identified, including 487 records from PubMed, 42 records from 


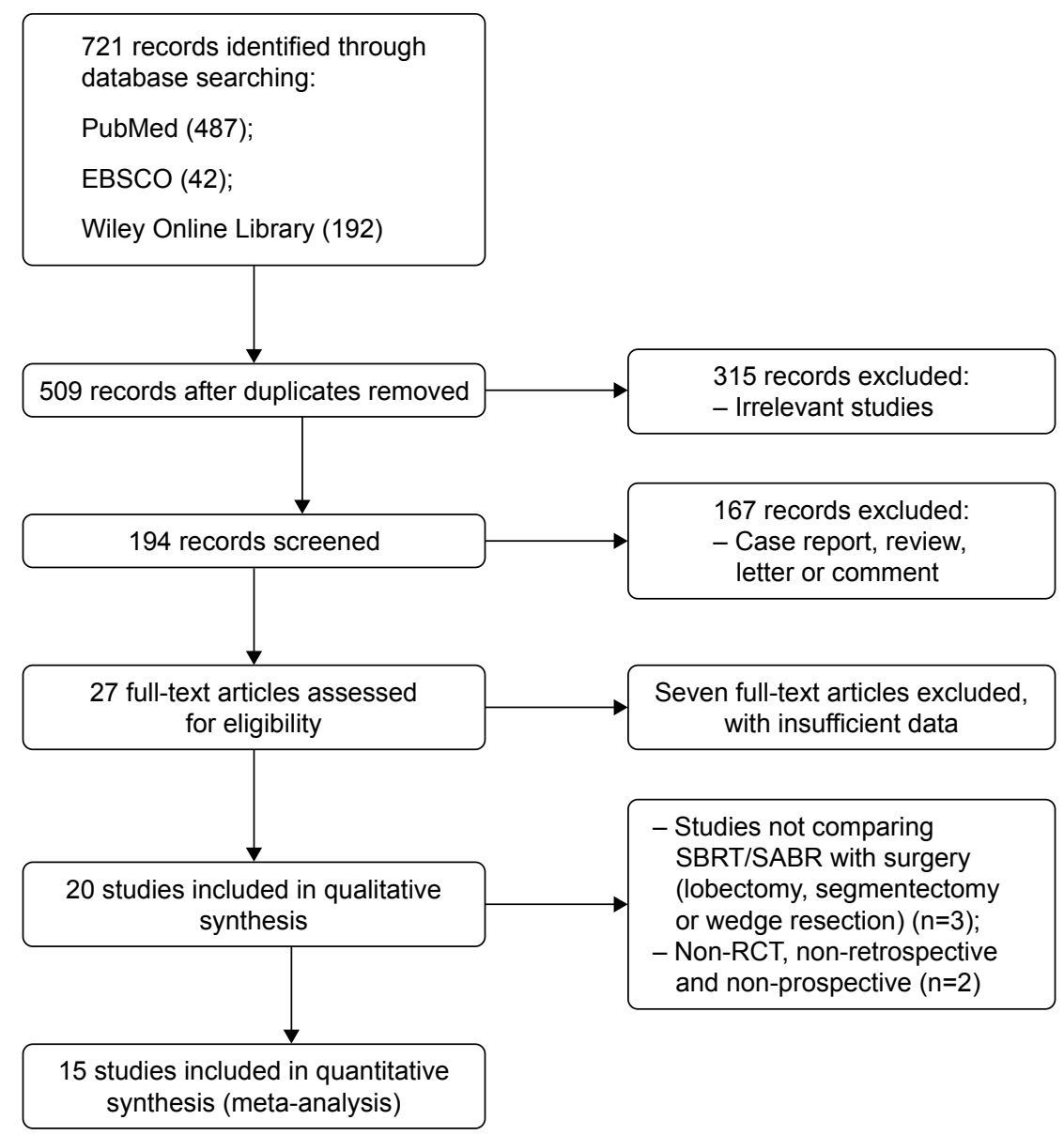

Figure I Flow diagram of the meta-analysis study selection process.

Abbreviations: SBRT, stereotactic body radiotherapy; SABR, stereotactic ablative radiotherapy; RCT, randomized controlled trial.

EBSCO, and 192 records from the Wiley Online Library. After excluding duplicates and irrelevant publications, 27 full-text studies were assessed for eligibility. Twenty studies were pooled into a qualitative synthesis. Further, we excluded five records that were not RCTs nor retrospective or prospective studies. Fifteen studies were included into the metaanalysis, including one RCT and 14 retrospective studies.

\section{Study characteristics}

Fifteen studies, reported between 2010 and 2016, were included in the final meta-analysis, including one RCT and 14 retrospective studies..$^{5-7,11-22}$ Among these studies, 11 articles used a propensity score or a matched pair method. ${ }^{5-7,11-14,17,19-21}$ Characteristics of the included studies are presented in Table 1. Seven studies were conducted in North America, four in Europe, three in Japan, and one in China. The sample size ranged from 58 to 3,562 . The median or mean age for the majority of the included studies ranged from 66 to 79 years. The mean/median size of tumor ranged from 16 to $30 \mathrm{~mm}$. In quality assessments, RCTs were evaluated according to seven items: randomization, blinding, concealed allocation, baseline features, eligibility criteria, loss to follow-up, and selection bias in the scores of assessing the NRSs by the NOS. The scores ranged from 5 to 7 , which indicated they represented the best evidence currently available.

\section{SBRT/SABR versus surgery}

In total, 15 articles compared SBRT/SABR and surgery, where five articles presented evidence on the superiority of SBRT/SABR, and non-significant survival difference was reported in nine studies. Only four studies showed that surgery is associated with a significantly better outcome. However, our analysis of the pooled data showed a significantly higher HR of SBRT/SABR versus surgery ( $\mathrm{HR}=1.40 ; 95 \%$ CI: $1.21,1.61 ; P<0.00001 ;$ Figure 2A). A significant medium heterogeneity was shown $\left(I^{2}=59 \%, P=0.002\right)$. A sensitivity analysis by excluding one individual record at a time did not detect a significant change in any study.

Six studies reported data on RFS. The HR comparing SBRT/SABR and surgery was 1.84 (95\%CI: 1.26, 2.68 ; $P=0.002$; Figure 2B). The pooled result showed that SBRT/ SABR increased the incidence of recurrence and the surgical 
Table I Basic characteristics of all of the pooled studies in the meta-analysis

\begin{tabular}{|c|c|c|c|c|c|c|c|c|c|c|}
\hline \multirow[t]{2}{*}{ Study } & \multirow[t]{2}{*}{ Year } & \multirow[t]{2}{*}{ Country } & \multirow[t]{2}{*}{$\begin{array}{l}\text { Study } \\
\text { design }\end{array}$} & \multirow[t]{2}{*}{$\begin{array}{l}\text { TNM (7th } \\
\text { edition) }\end{array}$} & \multicolumn{2}{|l|}{$\begin{array}{l}\text { Number of } \\
\text { patients }\end{array}$} & \multicolumn{2}{|c|}{$\begin{array}{l}\text { Age (mean } \pm S D \\
\text { or range) }\end{array}$} & \multicolumn{2}{|l|}{$\begin{array}{l}\text { Gender } \\
\text { (male/female) }\end{array}$} \\
\hline & & & & & SBRT/SABR & Surgery & SBRT/SABR & Surgery & SBRT/SABR & Surgery \\
\hline \multirow[t]{2}{*}{ Wang et $\mathrm{al}^{12}$} & 2016 & People's & RS & TINOMO & 35 & 35 & $77.1 \pm 5.2$ & $74.8 \pm 6.6$ & $33 / 2$ & $33 / 2$ \\
\hline & & Republic of China & & & & & & & & \\
\hline Rosen et al" & 2016 & USA & RS & $\mathrm{TI} / 2 \mathrm{aNOMO}$ & $|, 78|$ & $\mathrm{I}, 78 \mathrm{I}$ & $75.5 \pm 9.1$ & $74.8 \pm 7.8$ & $\mathrm{I}, 0 \mid 4 / 767$ & $\mathrm{I}, 004 / 777$ \\
\hline Ezer et $\mathrm{al}^{13}$ & 2015 & Canada and USA & RS & $\mathrm{TI} / 2 \mathrm{aNOMO}$ & 362 & $|, 88|$ & $78 \pm 6.5$ & $76 \pm 5.7$ & $127 / 235$ & $809 / 1,072$ \\
\hline Hamaji et al ${ }^{14}$ & 2015 & Japan & RS & $\mathrm{TI} / 2 \mathrm{aNOMO}$ & $4 I$ & 41 & $73(58-85)$ & $74(6 I-86)$ & $31 / 10$ & $32 / 9$ \\
\hline van den Berg et $\mathrm{al}^{15}$ & 2015 & the Netherlands & RS & $\mathrm{TI} / 2 \mathrm{aNOMO}$ & 197 & 143 & $77(52-93)$ & $67(40-84)$ & $143 / 54$ & $96 / 47$ \\
\hline Chang et $\mathrm{al}^{16}$ & 2015 & USA & RCT & $\mathrm{TI} / 2 \mathrm{aNOMO}$ & 31 & 27 & $67.1(43-82)$ & $66.7(5 \mathrm{I}-85)$ & $14 / 17$ & $11 / 16$ \\
\hline Kastelijn et al ${ }^{17}$ & 2015 & the Netherlands & RS & TI/2NOMO & 53 & 175 & $71.6 \pm 10.2$ & $66.5 \pm 9.1$ & $19 / 34$ & $109 / 66$ \\
\hline Nakagawa et al ${ }^{18}$ & 2014 & Japan & RS & $\mathrm{TI} / 2 \mathrm{aNOMO}$ & 35 & 183 & $79.8 \pm 2.8$ & $78.3 \pm 2.5$ & $25 / 10$ & $|22 / 6|$ \\
\hline Matsuo et al ${ }^{19}$ & 2014 & Japan & RS & $\mathrm{TI} / 2 \mathrm{aNOMO}$ & 53 & 53 & $76(58-86)$ & $76(50-88)$ & $42 / 11$ & $37 / 16$ \\
\hline Port et $\mathrm{al}^{20}$ & 2014 & USA & RS & TINOMO & 23 & 76 & $76(70-79)$ & $72(66-77)$ & $12 / 11$ & $26 / 50$ \\
\hline Crabtree et $\mathrm{al}^{5}$ & 2014 & USA & RS & $\mathrm{TI} / 2 \mathrm{aNOMO}$ & 56 & 56 & $70.0 \pm 8.1$ & $70.7 \pm 10.6$ & $29 / 27$ & $32 / 24$ \\
\hline Verstegen et $\mathrm{al}^{6}$ & 2013 & the Netherlands & RS & TI-3NOMO & 64 & 64 & $67.95 \pm 8.84$ & $70.53 \pm 9.91$ & $37 / 27$ & $36 / 28$ \\
\hline Varlotto et $\mathrm{al}^{21}$ & 2013 & USA & RS & $\mathrm{TI} / 2 \mathrm{NOMO}$ & 137 & 180 & 73.3 (5I-92) & $68.3(38-87)$ & $66 / 71$ & $99 / 81$ \\
\hline Palma et $\mathrm{al}^{7}$ & 2011 & the Netherlands & RS & TI/2NOMO & 60 & 60 & $79(76-8 I)$ & $79(76-80)$ & $40 / 20$ & $40 / 20$ \\
\hline Grills et a ${ }^{22}$ & 2010 & USA & RS & $\mathrm{TI} / 2 \mathrm{~N} 0 \mathrm{MO}$ & 58 & 69 & $74(69-78)$ & 78 (55-89) & $22 / 36$ & $28 / 4 \mid$ \\
\hline
\end{tabular}

Abbreviations: SD, standard deviation; SBRT, stereotactic body radiotherapy; SABR, stereotactic ablative radiotherapy; RS, retrospective study; RCT, randomized controlled trial; TNM, TNM classification of malignant tumors.

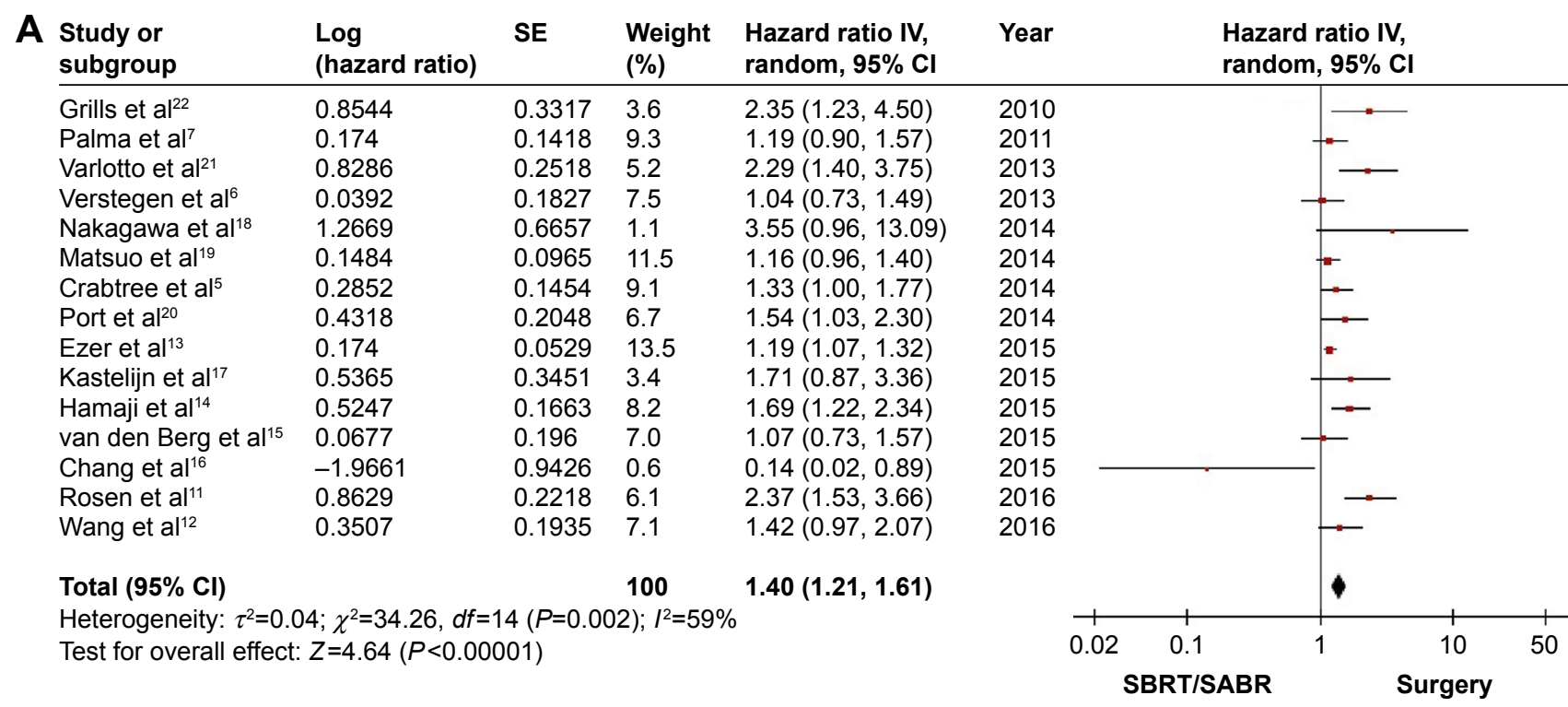

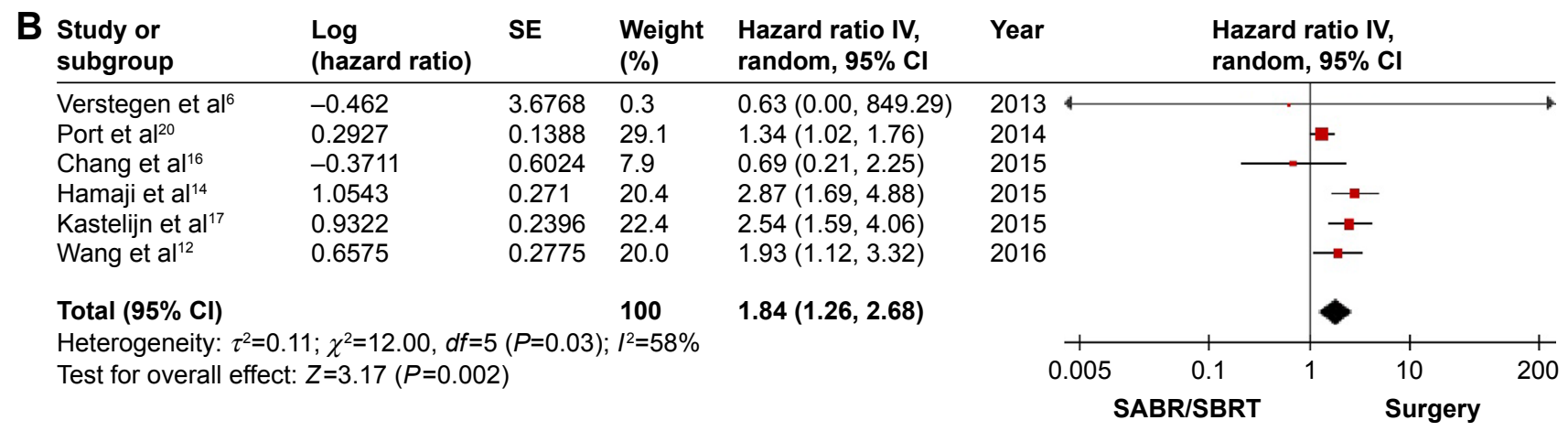

Figure 2 Forest plot of the survival for SBRT/SABR group versus surgical group. Including the OS (A) and the RFS (B).

Abbreviations: IV, inverse variance; Cl, confidence interval; SBRT, stereotactic body radiotherapy; SABR, stereotactic ablative radiotherapy; OS, overall survival; RFS, recurrence-free survival. 
group had a significantly longer RFS. A significant medium heterogeneity was shown $\left(I^{2}=58 \%, P=0.03\right)$. A sensitivity analysis by excluding one individual record at a time did not detect a significant change in any study. The funnel plot was symmetrical (Figure 3). Six studies reported data on the rate of locoregional recurrence. An analysis of the pooled data showed no significant difference between the SBRT/SABR group and surgical group $(\mathrm{HR}=1.17$; 95\% CI: 0.68, 1.98; $P=0.57$; Figure 4A). A significantly high heterogeneity was shown $\left(I^{2}=77 \%, P=0.001\right)$. A sensitivity analysis excluding one individual record at a time did not detect a significant change in any study.

Overall, five studies provided data on the rate of distant recurrence. Meta-analysis of the pooled data showed that there was no significant difference between SBRT/SABR and surgery $(\mathrm{HR}=1.36$; 95\% CI: 0.77, 2.39; $P=0.29$; Figure 4B). This meant that, compared with the SBRT/SABR, surgery did not have any significant advantage on distant recurrence. A significantly medium heterogeneity was shown $\left(I^{2}=69 \%, P=0.007\right)$. A sensitivity analysis excluding one individual record at a time did not detect a significant change in any study.

\section{SBRT/SABR versus lobectomy or sublobectomy}

Four articles provided available data of OS, comparing SBRT/ SABR (1,989 patients) and lobectomy (1,985 patients); five studies reported data on SBRT/SABR (510 patients) versus sublobectomy (2,096 patients); and three studies provided data on SBRT/SABR (440 patients) versus wedge resection (1,482 patients). However, data of RFS and the incidence of locoregional and distant recurrence was not available, and data of OS in segmentectomy was not provided in these studies separately. Meta-analysis of the

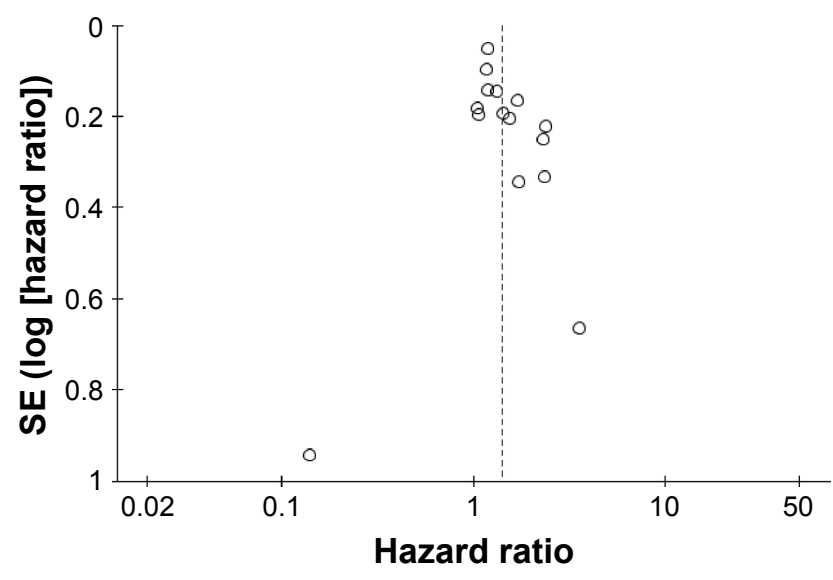

Figure 3 Results publication bias analysis - funnel plot. pooled data showed that there was a significant statistical difference in the OS between the SBRT/SABR group and the lobectomy group ( $\mathrm{HR}=1.46 ; 95 \% \mathrm{CI}: 1.03,2.06 ; P=0.03$; Figure 5A). Moreover, by comparing the OS between SBRT/ SABR and sublobectomy, an analysis showed that the surgical group also had a significant advantage (HR $=1.40 ; 95 \%$ CI: $1.09,1.80 ; P=0.008$; Figure 5B). Finally, a meta-analysis comparing SBRT/SABR versus wedge resection showed that there was a significant statistical difference in OS, with wedge resection being superior to SBRT/SABR ( $\mathrm{HR}=1.48$; 95\% CI: 1.01, 2.16; $P=0.04$; Figure 5C). Taken together, our results suggest that, compared with the SBRT/SABR group, both lobectomy and sublobectomy had significant advantages on OS for patients with T1-3N0M0 NSCLC. Heterogeneity is shown in the figures.

\section{Discussion}

In recent years, there are concerns about whether SBRT/ SABR or surgery is better for patients with T1-3N0M0 NSCLC. Furthermore, compared with SBRT/SABR, do different operative procedures have different efficacy? These have not been clarified thus far. As our results show, the studies pooled in our analyses indicated that there were no statistically significant differences between surgery and SBRT/SABR, probably due to limitations of sample. HRs in these studies seemed partial toward surgery. Only one research reported a pooled analysis of two randomized trials of SABR versus lobectomy for operable stage I NSCLC. ${ }^{16}$ In their findings, the estimated OS at 3 years was $95 \%$ (95\% CI: $85 \%-100 \%$ ) in the SABR group compared with $79 \%$ (95\% CI: 64\%-97\%) in the surgical group (HR $=0.14$, 95\% CI: $0.017,1.190 ; P=0.037)$. The RFS rate at 3 years was $86 \%$ (95\% CI: $74 \%-100 \%)$ in the SABR group and $80 \%$ (95\% CI: $65 \%-97 \%)$ in the surgical group (HR $=0.69$, $95 \%$ CI: $0.21,2.29 ; P=0.54)$. Although this study suggests that SABR may be better tolerated than surgery for operable clinical stage I NSCLC, it is limited by a small patient sample size $(\mathrm{n}=58)$ and short follow-up (median follow-up: SABR group, 40.2 months; surgery group, 35.4 months).${ }^{16}$ Nine retrospective studies suggested that clinical outcomes for patients with T1-3N0M0 NSCLC after SBRT/SABR were equal to that after surgery, and they also indicated that SBRT/ SABR may be better tolerated than surgery. ${ }^{5,17}$ However, in another two studies containing thousands of patients, results showed that surgery was associated with a significantly better outcome than SBRT/SABR. ${ }^{11,13}$ Consistently, in a nonrandomized population of patients selected for surgery versus SBRT/SABR, OS was higher in surgical patients. ${ }^{22}$ Although 


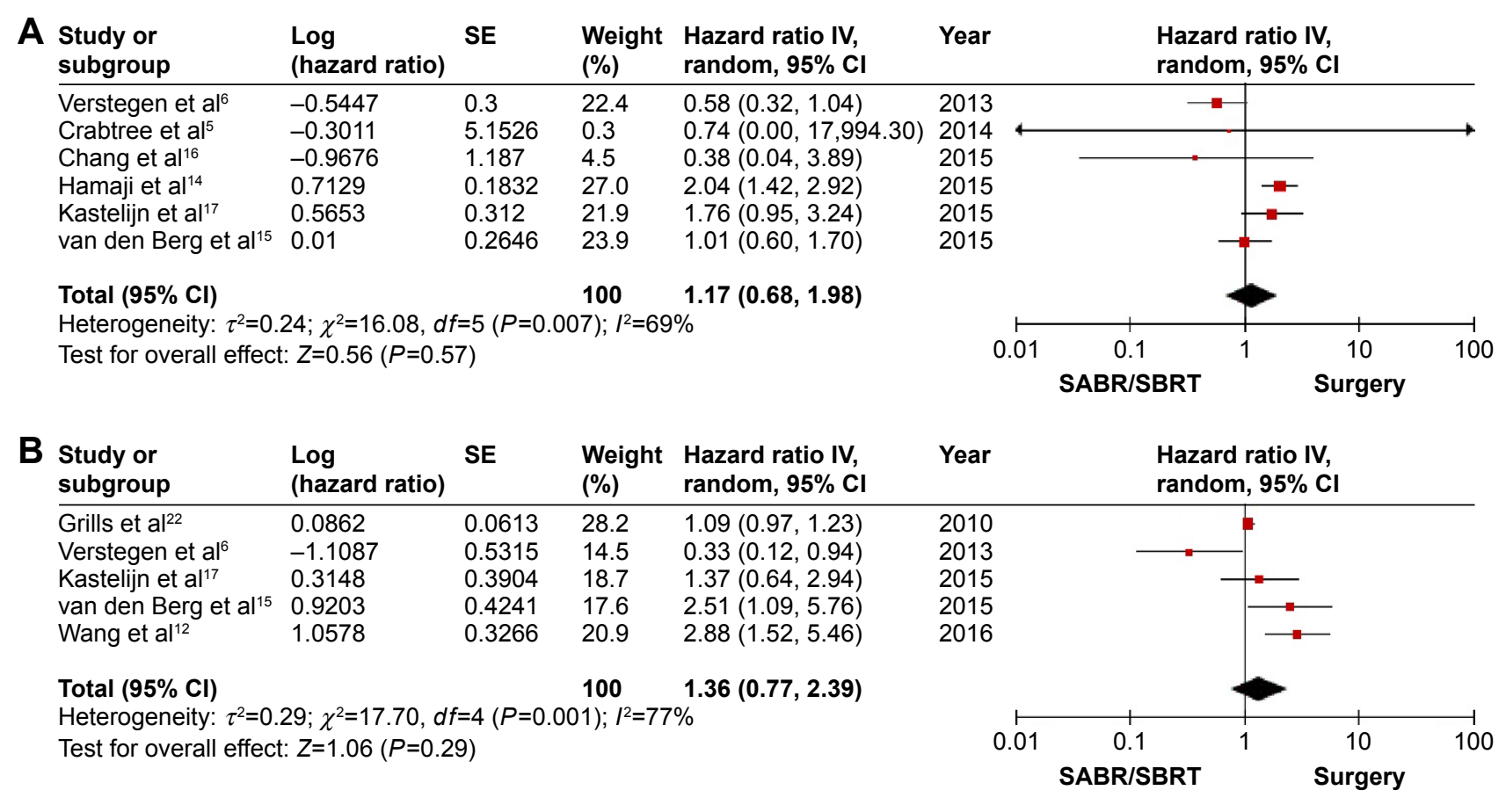

Figure 4 Forest plot of the incidence of recurrence for SBRT/SABR group versus surgical group, including (A) the incidence of locoregional recurrence and (B) the incidence of distant recurrence.

Abbreviations: IV, inverse variance; Cl, confidence interval; SBRT, stereotactic body radiotherapy; SABR, stereotactic ablative radiotherapy.

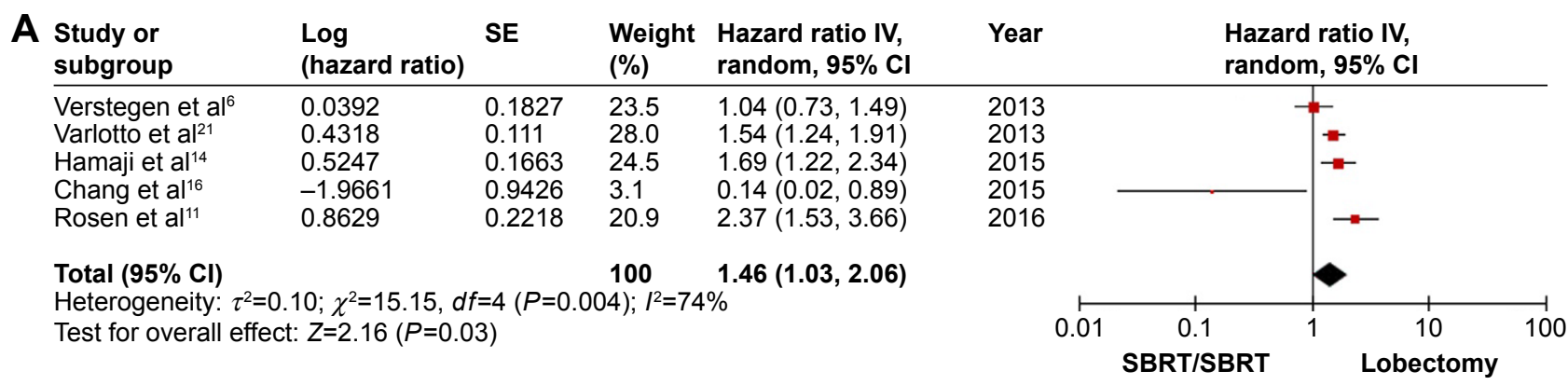

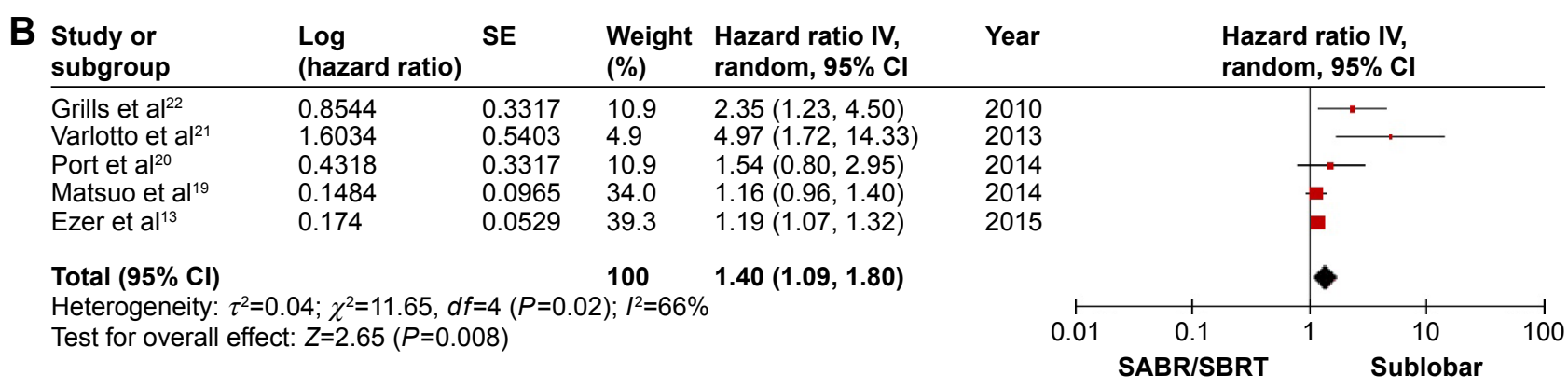

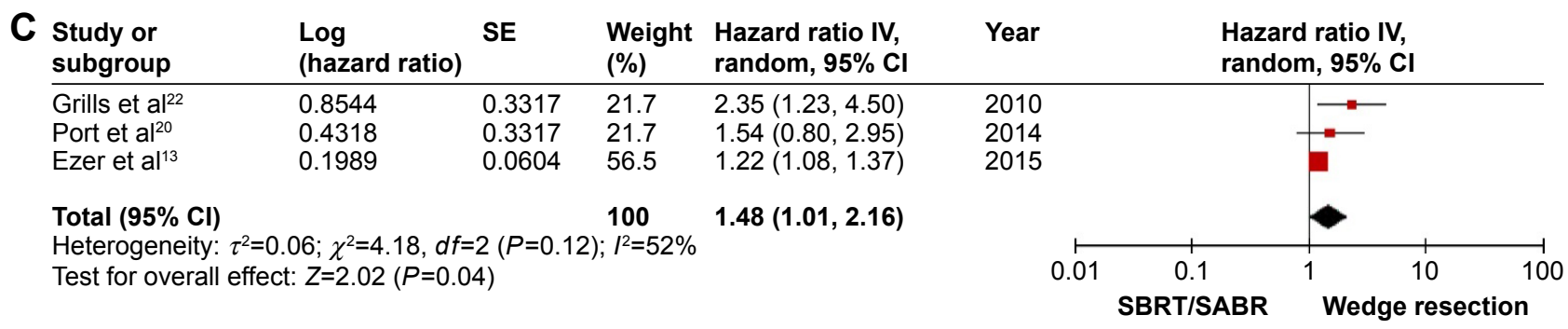

Figure 5 Forest plot of the OS for SBRT/SABR group versus different surgical groups, including (A) the OS for SBRT/SABR group versus lobectomy, (B) the OS for SBRT/ SABR group versus sublobectomy, and $(\mathbf{C})$ the OS for SBRT/SABR group versus wedge resection.

Abbreviations: IV, inverse variance; Cl, confidence interval; SBRT, stereotactic body radiotherapy; SABR, stereotactic ablative radiotherapy; OS, overall survival. 
the surgical patients were more often male, younger, with a better performance score and pulmonary function than those treated by SBRT/SABR, SBRT/SABR patients often had smaller tumors. ${ }^{17}$ In our pooled articles, most studies used a propensity score or case-control matching to attenuate bias caused by the variations of the clinical baseline.

Finally, our meta-analyses displayed that surgery, both lobectomy and sublobectomy, have advantages in outcomes when undertaken prior to SBRT/SABR. In a study by Hamaji et al, results suggested that video-assisted thoracic surgery (VATS) may offer more significant favorable long-term outcomes compared to SBRT/SABR in patients with clinical stage I NSCLC. ${ }^{14}$ Meanwhile, another study found that SBRT/SABR conferred worse locoregional tumor control, as a result of more nodal failures compared with surgery. ${ }^{15}$ Furthermore, Matsuo et al suggested that surgery may be superior to SBRT for tumor size $>20 \mathrm{~mm}$ in diameter, whereas equivalent outcomes can be expected when the size was $<20 \mathrm{~mm} .{ }^{19}$ Tumor size has not been confirmed in the present study because of data unavailability; however, in our meta-analysis, results suggested that SBRT/SABR had a worse OS and RFS than surgery for patients with T1-3N0M0 NSCLC. Some studies showed that SBRT/SABR might be better tolerated than surgery. ${ }^{5,13,19}$ Nowadays, with the progress of modern thoracic surgery such as VATS, endurance to surgery has been enhanced considerably. Most pulmonary lobectomies and sublobectomies were performed with VATS and resulted in satisfactory 5-year OS rates and lower complication rates in clinical practice. ${ }^{23,24}$ Our results show that sublobectomy, including wedge resection, has better survival prior to SBRT/SABR, whereas sublobectomy may be associated with fewer complications, better postoperative lung function, and tolerance, which is limited to appropriate patients. Therefore, our results suggest surgical treatment should be considered for potentially operable patients if possible.

Undoubtedly, SBRT/SABR has several inherent advantages for some specific patients such as: shorter treatment times, favorable side effect profile, ease of being undertaken in an outpatient setting, and rarely requiring hospitalization. Nowadays, for patients with T1-3N0M0 NSCLC, the choice of treatment, surgical resection or SBRT/SABR, is made based on multiple variables such as patient wishes and tumor characteristics, TNM classification, pathological classification, and local practice. Considering current studies, although many pooled studies were retrospective, they represented the best evidence currently available.

Admittedly, there were several limitations in this study: 1) RCTs, prospective studies, and retrospective studies were assessed by different standards and pooled together. 2) Indirect data acquisition methods were used, such as when extracting data from the survival curves for meta-analysis. 3) More detailed subgroups analyses are not shown, because of the paucity of data. 4) Many of the included studies were retrospective, and more RCTs are needed.

\section{Conclusion}

In conclusion, our meta-analysis provides evidence that surgery may be more preferable than SBRT/SABR, with regard to OS, RFS, and locoregional/distant recurrence rate. Both lobectomy and sublobectomy may offer a more favorable OS. Our results indicate that patients with T1-3N0M0 NSCLC should preferably be treated by surgery prior to SBRT/SABR.

\section{Acknowledgments}

This work was supported by the National Natural Science Foundation of China (Grant Nos 81370587, 81401875, 8167100185; www.nsfc.gov.cn/), Training Programme for the Talents of Zhongshan Hospital, Fudan University (Grant No 2015ZSYXGG03), Zhengyi Scholar Foundation of School of Basic Medical Sciences, Fudan University (Grant No S18-04; http://medicine.fudan.edu.cn/), FDUROP (Fudan's Undergraduate Research Opportunities Program; Grant No 15016), and National University Student Innovation Program (Grant No 15016) (www.fdurop.fudan.edu.cn/). The authors would like to thank Prof. Yuezhen Dai (College of Foreign Languages and Literatures, Fudan University) and the International Science Editing Co. for editing the manuscript.

\section{Disclosure}

The authors report no conflicts of interest in this work.

\section{References}

1. Siegel RL, Miller KD, Jemal A. Cancer statistics, 2015. CA Cancer J Clin. 2015;65(1):5-29.

2. Bertolaccini L, Terzi A, Ricchetti F, Alongi F. Surgery or stereotactic ablative radiation therapy: how will be treated operable patients with early stage not small cell lung cancer in the next future? Ann Transl Med. 2015;3(2):25.

3. Khullar OV, Liu Y, Gillespie T, et al. Survival after sublobar resection versus lobectomy for clinical stage IA lung cancer: an analysis from the National Cancer Data Base. J Thorac Oncol. 2015;10(11): 1625-1633.

4. Boily G, Filion É, Rakovich G, et al; Comité de l'évolution des pratiques en oncologie. Stereotactic ablative radiation therapy for the treatment of early-stage non-small-cell lung cancer: CEPO review and recommendations. J Thorac Oncol. 2015;10(6):872-882.

5. Crabtree TD, Puri V, Robinson C, et al. Analysis of first recurrence and survival in patients with stage I non-small cell lung cancer treated with surgical resection or stereotactic radiation therapy. J Thorac Cardiovasc Surg. 2014;147(4):1183-1191; discussion 1191-1192. 
6. Verstegen NE, Oosterhuis JW, Palma DA, et al. Stage I-II non-smallcell lung cancer treated using either stereotactic ablative radiotherapy (SABR) or lobectomy by video-assisted thoracoscopic surgery (VATS): outcomes of a propensity score-matched analysis. Ann Oncol. 2013; 24(6):1543-1548

7. Palma D, Visser O, Lagerwaard FJ, Belderbos J, Slotman B, Senan S. Treatment of stage I NSCLC in elderly patients: a population-based matched-pair comparison of stereotactic radiotherapy versus surgery. Radiother Oncol. 2011;101(2):240-244.

8. Vansteenkiste J, Crinò L, Dooms C, et al. 2nd ESMO Consensus Conference on Lung Cancer: early-stage non-small-cell lung cancer consensus on diagnosis, treatment and follow-up. Ann Oncol. 2014;25(8): 1462-1474.

9. Higgins JP, Thompson SG, Deeks JJ, Altman DG. Measuring inconsistency in meta-analyses. BMJ. 2003;327(7414):557-560.

10. Tierney JF, Stewart LA, Ghersi D, Burdett S, Sydes MR. Practical methods for incorporating summary time-to-event data into meta-analysis. Trials. 2007;8:16.

11. Rosen JE, Salazar MC, Wang Z, et al. Lobectomy versus stereotactic body radiotherapy in healthy patients with stage I lung cancer. J Thorac Cardiovasc Surg. 2016;152(1):44-54.e9.

12. Wang $P$, Zhang D, Guo XG, et al. A propensity-matched analysis of surgery and stereotactic body radiotherapy for early stage non-small cell lung cancer in the elderly. Medicine (Baltimore). 2016;95(52):e5723.

13. Ezer N, Veluswamy RR, Mhango G, Rosenzweig KE, Powell CA, Wisnivesky JP. Outcomes after stereotactic body radiotherapy versus limited resection in older patients with early-stage lung cancer. J Thorac Oncol. 2015;10(8):1201-1206.

14. Hamaji M, Chen F, Matsuo Y, et al. Video-assisted thoracoscopic lobectomy versus stereotactic radiotherapy for stage I lung cancer. Ann Thorac Surg. 2015;99(4):1122-1129.

15. van den Berg LL, Klinkenberg TJ, Groen HJ, Widder J. Patterns of recurrence and survival after surgery or stereotactic radiotherapy for early stage NSCLC. J Thorac Oncol. 2015;10(5):826-831.
16. Chang JY, Senan S, Paul MA, et al. Stereotactic ablative radiotherapy versus lobectomy for operable stage I non-small-cell lung cancer: a pooled analysis of two randomised trials. Lancet Oncol. 2015;16(6): 630-637.

17. Kastelijn EA, El Sharouni SY, Hofman FN, et al. Clinical outcomes in early-stage NSCLC treated with stereotactic body radiotherapy versus surgical resection. Anticancer Res. 2015;35(10):5607-5614.

18. Nakagawa T, Negoro Y, Matsuoka T, Okumura N, Dodo Y. Comparison of the outcomes of stereotactic body radiotherapy and surgery in elderly patients with cT1-2N0M0 non-small cell lung cancer. Respir Investig. 2014;52(4):221-226.

19. Matsuo Y, Chen F, Hamaji M, et al. Comparison of long-term survival outcomes between stereotactic body radiotherapy and sublobar resection for stage I non-small-cell lung cancer in patients at high risk for lobectomy: a propensity score matching analysis. Eur J Cancer. 2014; 50(17):2932-2938.

20. Port JL, Parashar B, Osakwe N, et al. A propensity-matched analysis of wedge resection and stereotactic body radiotherapy for early stage lung cancer. Ann Thorac Surg. 2014;98(4):1152-1159.

21. Varlotto J, Fakiris A, Flickinger J, et al. Matched-pair and propensity score comparisons of outcomes of patients with clinical stage I nonsmall cell lung cancer treated with resection or stereotactic radiosurgery. Cancer. 2013;119(15):2683-2691.

22. Grills IS, Mangona VS, Welsh R, et al. Outcomes after stereotactic lung radiotherapy or wedge resection for stage I non-small-cell lung cancer. J Clin Oncol. 2010;28(6):928-935.

23. Shen Y, Wang H, Feng M, Xi Y, Tan L, Wang Q. Single-versus multipleport thoracoscopic lobectomy for lung cancer: a propensity-matched study†. Eur J Cardiothorac Surg. 2016;49(Suppl 1):i48-i53.

24. Lin Z, Xi J, Xu S, Jiang W, Wang L, Wang Q. Uniportal video-assisted thoracic surgery lobectomy in semiprone position: primary experience of 105 cases. J Thorac Dis. 2015;7(12):2389-2395.
OncoTargets and Therapy

\section{Publish your work in this journal}

OncoTargets and Therapy is an international, peer-reviewed, open access journal focusing on the pathological basis of all cancers, potential targets for therapy and treatment protocols employed to improve the management of cancer patients. The journal also focuses on the impact of management programs and new therapeutic agents and protocols on

\section{Dovepress}

patient perspectives such as quality of life, adherence and satisfaction The manuscript management system is completely online and includes a very quick and fair peer-review system, which is all easy to use. Visit http://www.dovepress.com/testimonials.php to read real quotes from published authors. 